\title{
Interfacial Spin-Orbit Coupling: A Platform for Superconducting Spintronics
}

\author{
Isidoro Martínez, ${ }^{1}$ Petra Högl, ${ }^{2}$ César González-Ruano, ${ }^{1}$ Juan Pedro Cascales, ${ }^{3}$ Coriolan Tiusan, ${ }^{4,5}$ \\ Yuan Lu, ${ }^{5}$ Michel Hehn, ${ }^{5}$ Alex Matos-Abiague, ${ }^{6}$ Jaroslav Fabian, ${ }^{2}$ Igor Žutić,,${ }^{2,7, *}$ and \\ Farkhad G. Aliev@ ${ }^{1, \dagger}$ \\ ${ }^{1}$ Departamento Física de la Materia Condensada C-III, Instituto Nicolás Cabrera and Condensed Matter Physics \\ Institute (IFIMAC), Universidad Autónoma de Madrid, 28049 Madrid, Spain \\ ${ }^{2}$ Institute for Theoretical Physics, University of Regensburg, 93040 Regensburg, Germany \\ ${ }^{3}$ Francis Bitter Magnet Laboratory, Massachusetts Institute of Technology, Cambridge, Massachusetts \\ 02139, USA \\ ${ }^{4}$ Department of Physics and Chemistry, Center of Superconductivity Spintronics and Surface Science C4S, \\ Technical University of Cluj-Napoca, 400114 Cluj-Napoca, Romania \\ ${ }^{5}$ Institut Jean Lamour, Nancy Universite, 54506 Vandoeuvre-les-Nancy Cedex, France \\ ${ }^{6}$ Department of Physics and Astronomy, Wayne State University, Detroit, Michigan 48201, USA \\ ${ }^{7}$ Department of Physics, University at Buffalo, State University of New York, Buffalo, New York 14260, USA
}

(Received 5 December 2018; revised manuscript received 4 November 2019; published 17 January 2020)

Spin-orbit coupling (SOC) is a key interaction in spintronics, allowing electrical control of spin or magnetization and, vice versa, magnetic control of electrical current. However, recent advances have revealed much broader implications of SOC that is also central to the design of topological states with potential applications from low-energy dissipation and faster magnetization switching to high tolerance of disorder. SOC and the resulting emergent interfacial spin-orbit fields are simply realized in junctions through structural inversion asymmetry, while the anisotropy in magnetoresistance (MR) allows their experimental detection. Surprisingly, we demonstrate that an all-epitaxial ferromagnet $/ \mathrm{MgO} / \mathrm{metal}$ junction with a single ferromagnetic region and only negligible MR anisotropy undergoes a remarkable transformation below the superconducting transition temperature of the metal. The superconducting junction has a MR anisotropy 3 orders of magnitude higher and could enable novel applications in superconducting spintronics. In contrast to common realizations of MR effects that require a finite applied magnetic field, our system is designed to have two stable zero-field states with mutually orthogonal magnetizations: in plane and out of plane. This bistable magnetic anisotropy allows us to rule out orbital and vortex effects due to an applied magnetic field and identify the SOC origin of the observed MR. Such MR reaches approximately $20 \%$ without an applied magnetic field and could be further increased for large magnetic fields that support vortices. Our findings call for a revisit of the role of SOC, even when it seems negligible in the normal state, and suggest an alternative platform for superconducting spintronics.

DOI: 10.1103/PhysRevApplied.13.014030

\section{INTRODUCTION}

For more than 150 years magnetoresistive effects have provided attractive platforms to study spin-dependent phenomena and enable key spintronic applications [1]. Primarily, spintronics relies on junctions with at least two ferromagnetic layers to provide sufficiently large magnetoresistance (MR). Record room-temperature MR is obtained with and commercial applications use magnetic tunnel junctions (MTJs) of common ferromagnets, such as $\mathrm{Co}$ and $\mathrm{Fe}$ with a $\mathrm{MgO}$ tunnel barrier [2,3]. Alternatively,

\footnotetext{
*zigor@buffalo.edu

†farkhad.aliev@uam.es
}

MR occurs in single ferromagnetic layers with an interplay of interfacial spin-orbit coupling (SOC). However, in metallic systems this phenomenon, known as "tunneling anisotropic MR" (TAMR) [4], is typically less than $1 \%$ and precludes practical applications. Here we show experimentally that a negligible $\mathrm{MR}$ in an all-epitaxial ferromagnet $/ \mathrm{MgO} /$ metal junction is drastically enhanced below the superconducting transition temperature of the metal. We find that this peculiar behavior with the role of the interfacial SOC supports the formation of spin-triplet superconductivity, which could expand the range of applications in superconducting spintronics [5-7] by better integrating superconductivity and ferromagnetism. Building on our experience in fabricating high-quality all-epitaxial 

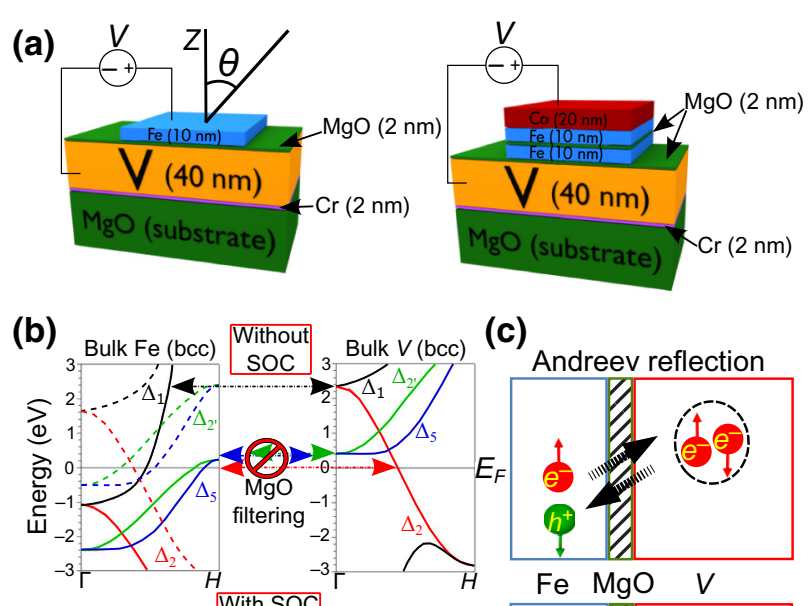

(c)
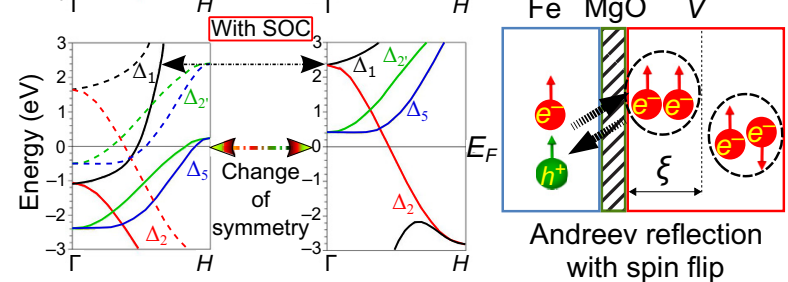

Andreev reflection with spin flip

FIG. 1. Junction geometry, electronic structure, and Andreev reflection. (a) $\mathrm{Fe} / \mathrm{MgO} / \mathrm{V}$ and $\mathrm{Co} / \mathrm{Fe} / \mathrm{MgO} / \mathrm{Fe} / \mathrm{MgO} / \mathrm{V}$ junctions, where $\mathrm{Co} / \mathrm{Fe}$ forms a hard ferromagnet. $\theta$ is the angle between the magnetization, $\mathbf{M}$, and the interface normal. (b) First-principles electronic structure calculations illustrate the orbital-symmetry-controlled tunneling across $\mathrm{Fe} / \mathrm{MgO} / \mathrm{V}$ junctions without (with) spin-orbit coupling in the upper (lower) panel, where $\Delta_{1}$ and $\Delta_{5}$ are different orbital symmetries. For $\mathrm{Fe}(100)$, majority (minority) bands are given by solid (dashed) lines. (c) Andreev reflection at the $\mathrm{Fe} / \mathrm{MgO} / \mathrm{V}$ interface without (with) spin-flip scattering in the upper (lower) panel. The arrows denote the spin direction of the electrons $\left(e^{-}\right)$and holes $\left(h^{+}\right)$, black circles mark the resulting formation of Cooper pairs, and $\xi$ is the coherence length.

MgO-based MTJs [8,9], we design competing magnetic anisotropies that allow multiple magnetic configurations even at zero applied magnetic field. Furthermore, in our all-epitaxial junctions (see Fig. 1) the role of SOC is more pronounced as it avoids interfacial wave-vector averaging, which is common to other superconducting junctions.

The quest for spin-triplet superconductivity in ferromagnet/superconductor $(F / S)$ junctions was initially motivated by the long-range proximity effects to overcome the usual competition between superconductivity and ferromagnetism since spin-singlet superconductivity is strongly suppressed by the exchange field [10-12]. Such longrange triplet (LRT), with length scales expected only for normal-metal $(N) / S$ junctions, supports dissipationless spin currents that are important for emerging applications in superconducting spintronics [5-7,13-16]. While largeMR behavior can occur in superconducting junctions with only spin-singlet superconductivity, the presence of spintriplet supercurrents could also generate spin torque and alter the magnetic configuration or act as a phase battery $[6,16]$.

Unlike the common expectation that large MR implies multiple ferromagnet regions [6], while long-range triplet requires complex ferromagnet multilayers, typically relying on noncollinear or spiral magnetization (M) $[10,11,17]$ or half-metals $[5,18,19]$, we demonstrate that a collinear magnetization in a single ferromagnet layer provides both large MR and can support long-range triplet since it is accompanied by intrinsic interfacial scattering that mixes spin-singlet and spin-triplet pairing [20-22]. A zero-field MR of approximately $20 \%$ is further enhanced in large applied magnetic fields due to vortex formation. In an allepitaxial $F /$ insulator/ $S(F / I / S)$ junction, we realize a versatile building block for superconducting spintronics that is compatible with commercial spintronics based on $\mathrm{Fe} / \mathrm{MgO}$ junctions $[2,3,23]$. We focus on two types of junctions, shown in Fig. 1(a): (i) $\mathrm{Fe}(100) / \mathrm{MgO}(100) / \mathrm{V}(100)$ and (ii) $F 1 / I / F 2 / I / S$ : $\mathrm{Co} / \mathrm{Fe} / \mathrm{MgO}(100) / \mathrm{Fe}(100) / \mathrm{MgO}(100) / \mathrm{V}$ (100), where vanadium (V) becomes a superconductor below the critical temperature, $T_{C}=4 \mathrm{~K}$, while in the latter case magnetically hard $F 1(\mathrm{Co} / \mathrm{Fe})$ and soft $F 2$ (Fe) regions provide versatile control of the magnetization orientation. These systems illustrate the concept of proximitized materials [24] to design emergent properties that are absent in any constituent region of the junctions considered.

\section{EXPERIMENTAL DETAILS}

The normal state transport of these epitaxial junctions, with crystalline $\mathrm{MgO}$ and the conserved wave vector parallel to the interfaces, $\mathbf{k}_{\|}=0$, can be understood from the orbital-symmetry-controlled tunneling [25] across $\mathrm{Fe} / \mathrm{MgO} / \mathrm{V}$ shown in Fig. 1(b), obtained by fullpotential calculations with the WIEN2k code [26]. In view of the thickness of the $\mathrm{MgO}$ barrier, the main contribution to the tunneling comes from normal incidence at the $\mathbf{k}_{\|}=0$ ( $\Gamma$ point). Our $a b$ initio calculations show that at the Fermi level, $E_{F}$, the electron states are dominated by different orbital symmetries, $\Delta_{1}$ in Fe and $\Delta_{2}$ in $\mathrm{V}$, which, in the absence of interfacial scattering that disrupts symmetry, would yield zero low-bias conductance across $\mathrm{MgO}(100)$. Experimentally, in $\mathrm{Fe} / \mathrm{MgO} / \mathrm{V}$ such conductance does not vanish, but is about $10^{2}$ times smaller than in $\mathrm{Co} / \mathrm{Fe} / \mathrm{MgO} / \mathrm{Fe}$ and $\mathrm{Fe} / \mathrm{MgO} / \mathrm{Au}$ control samples with similar barrier quality and thickness, as well as similar lateral sizes. This finite low-bias conductance is consistent with the interfacial scattering and the structural inversion asymmetry of a junction $[1,4]$, which is responsible for the change of symmetry across $\mathrm{MgO}$ by relaxing the symmetry selection rules. From the absence of symmetry-enforced spin filtering for the $\mathrm{Fe} / \mathrm{MgO} / \mathrm{Au}$ junction, with see that the suppression of conductance in $\mathrm{Fe} / \mathrm{MgO} / \mathrm{V}$ is dominated by spin filtering rather than by the barrier strength, which 
is largely unchanged. While fabricated $\mathrm{Fe} / \mathrm{MgO}$ junctions are not strictly half-metallic, through $\Delta_{1}$ symmetry they provide a very large tunneling spin polarization $(85 \%$ in Ref. [2]), which is also the origin of the huge tunneling MR (TMR) in Fe/MgO-based MTJs [2,3].

The MTJ multilayer stacks are grown by molecularbeam epitaxy on $\mathrm{MgO}(100)$ single-crystal substrates in a chamber with a base pressure of $5 \times 10^{-11}$ mbar following the procedure described in Ref. [27]. First, a 10-nmthick-seed $\mathrm{MgO}$ antidiffusion underlayer is grown on the substrate to trap the residual $\mathrm{C}$. Then, the $\mathrm{V}$ electrode is deposited at room temperature and further annealed for flattening at $T>500^{\circ} \mathrm{C}$. The choice of the temperature and annealing time is monitored by RHEED-pattern analysis. Then the $\mathrm{MgO}$ insulating barrier layer is epitaxially grown to 2-nm thickness by electron-beam evaporation, precisely controlled at the submonolayer scale, by in situ RHEED intensity analysis. This $\mathrm{MgO}$ thickness is in the asymptotic transport regime [28], where the orbital symmetry filtering across the barrier leads to a main contribution to the $\mathbf{k}_{\|}=0$ tunneling. For a given thickness, the $\mathrm{MgO}$ barrier is typically not as strong as an aluminum oxide barrier. In our epitaxially grown $\mathrm{MgO}$ there is an additional reduction of the effective barrier to approximately $0.3-0.4 \mathrm{eV}$ for low-bias transport, which is consistent with transport measurements on epitaxial $\mathrm{Fe} / \mathrm{MgO}$-based MTJs reported in Ref. [3]. In contrast, the barrier is approximately $1 \mathrm{eV}$ higher for the polycrystalline nonepitaxial $\mathrm{Fe} / \mathrm{MgO}$-based MTJs studied in Ref. [2].

The epitaxial growth sequence is continued for all the other layers, leading to single-crystal MTJ stacks in which crystalline symmetry across the stack ensures the symmetry and $\mathbf{k}_{\|}$conservation of the Bloch electron function, allowing very good agreement between theory and experiment. For $F 1 / I / F 2 / I / S$ junctions [Fig. 1(a), right], the hard ferromagnet $F 1$ is formed from a 10 -nm-thick Fe layer, epitaxially grown on top of $\mathrm{MgO}$, and a $20-\mathrm{nm}$ Co layer on top of it. After growth by molecular-beam epitaxy, all the MTJ multilayer stacks are patterned in micrometer-sized square junctions by UV lithography and Ar-ion etching, controlled step-by-step in situ by Auger spectroscopy. Details of conductance measurements and the vector magnetic field control are given in Refs. [23,29]. A unique feature of our devices is the control of the remanent-magnetization direction of the magnetically soft electrode interfacing with $S$. Such control is possible due to the presence of competing perpendicular and in-plane magnetic anisotropies in 10-nm-thick epitaxial $\mathrm{Fe}$ layers interfacing with $\mathrm{MgO}$ [29].

\section{RESULTS}

The transport in the superconducting state is distinguished by the Andreev reflection, providing the microscopic mechanism for proximity-induced superconductivity $[1,10,11]$. During conventional Andreev reflection shown in Fig. 1(c) (upper panel), an electron is reflected backwards and converted into a hole with opposite charge and spin. This implies the doubling of the normal-state conductance [30] since two electrons are transferred across the interface into the superconductor region, where they form a spin-singlet Cooper pair. With unequal contributions of minority and majority spins in the ferromagnet region, not all electrons can find the partner of opposite spin to undergo Andreev reflection. Therefore, the spin polarization of the ferromagnet region can be studied through the suppression of Andreev reflection and the resulting low-bias conductance. While this procedure usually relies on point contacts [31], it was also applied for $F / I / S$ tunnel junctions [32] with additional suppression of Andreev reflection due to potential scattering at an insulator. In both cases, it is important to decouple the role of conductance suppression due to spin polarization from that arising from interfacial scattering [33-37]. In contrast to this conventional Andreev reflection, a spin-active interface with interfacial spin-flip scattering can also yield Andreev reflection with an equal spin of electrons and holes [38], which is responsible for a spin-triplet Cooper pair as shown in Fig. 1(c) (lower panel). Since Andreev reflection is the origin of superconducting proximity effects $[1,10-12]$, one expects that the resulting interfacial spintriplet correlations decay away from the interface both inside the superconductor region and inside the ferromagnetic region. The decay length inside the ferromagnet region is longest for spin-triplet (equal-spin) correlations $[6,7,10,11]$.

While the bias-dependent conductance, $G(V)$, can indicate the presence of interfacial SOC, similar $G(V)$ also arises from the k-independent interfacial spin-flip scattering $[22,38]$ due to local exchange coupling. Instead, a unique fingerprint of interfacial SOC is the magnetic anisotropy of $G(V)$. Within the normal state, $T>T_{C}$ or, equivalently, for bias above the superconducting gap $V \gg \Delta$, the TAMR in $F / I / N$ junctions can reveal such SOC through M-dependent $G$ [4]. This can be seen in Fig. 2, where the angle-dependent conductance, $G(\theta)$, is expressed using $\theta$ from Fig. 1(a). However, the magnitude of the observed TAMR, $[G(0)-G(\pi / 2)] / G(\pi / 2) \sim$ $0.01 \%$, with $G(\theta)$, while reproducible, is too small for any practical use.

Motivated by the prediction that MR can be enhanced in the superconducting state [22], we explore in the same junction the resulting $F / I / S$ conductance anisotropy. The corresponding out-of-plane magnetoanisotropic Andreev reflection (MAAR) [22] is the superconducting analog of the TAMR, defined as

$$
\operatorname{MAAR}=\frac{G(0)-G(\theta)}{G(\theta)},
$$



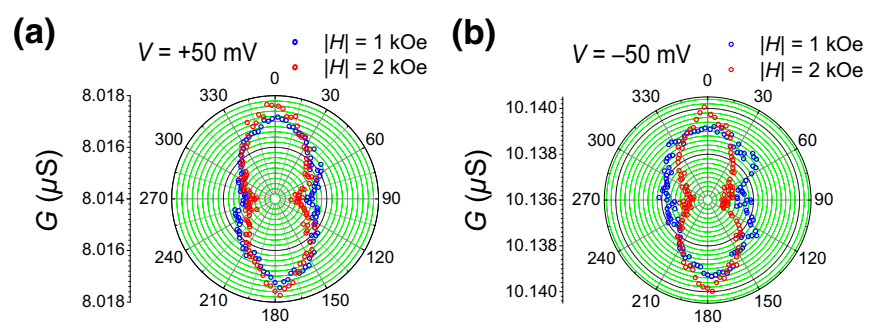

FIG. 2. Angular dependence of conductance for an out-ofplane rotation of magnetization $\mathbf{M}$. The conductance is measured for $\mathbf{M}$ at a polar angle $\theta$ normal to the interface at $T=0.3 \mathrm{~K}$ and applied bias $V=+50 \mathrm{mV}$ (a) and $V=-50 \mathrm{mV}$ (b) and applied fields $H=1 \mathrm{kOe}$ and $H=2 \mathrm{kOe}$. Since the voltage greatly exceeds $\Delta$ for vanadium, the resulting magnetic anisotropy can be used to extract the magnitude of normal-state TAMR, approximately $0.01 \%$ for $H=1 \mathrm{kOe}$ and approximately $0.02 \%$ for $H=2 \mathrm{kOe}$.

where $\theta$ is the magnetization angle with the interface normal (see Appendix A, which compares TAMR and MAAR). Useful reference information about the transport across the junction is provided in Fig. 3(a) by comparing a typical zero-field $G(V)$ below and above $T_{C}$ for $T=0.3 \mathrm{~K}$ $\left(T / T_{C}=0.08\right)$ and $T=10 \mathrm{~K}$, respectively. Just as in a widely used one-dimensional Blonder-Tinkham-Klapwijk (BTK) model for $G(V)$ of $N / I / S$ junctions, excluding SOC and the spin polarization [30], a common description generalizing it to $F / I / S$ junctions [22,31-34] relies on a dimensionless interfacial barrier parameter $Z$ (see Appendix F). The normal-state transparency for the BTK model, $1 /\left(1+Z^{2}\right)$, establishes a simple parameterization for the perfectly transparent junction with $Z=0$ and the tunnel junction with $Z \gg 1$ with vanishing transparency. The lowering of conductance is thus directly connected to the interfacial barrier [30]. The normalized conductance is consistent with an intermediate junction transparency (see Appendix F). It is lower than for point contacts, where the Andreev reflection is used to probe the spin polarization of ferromagnets $[31,33]$, but higher than for typical spinpolarized quasiparticle tunneling in $F / I / S$ junctions $[1,2]$. In the latter case [32], seen also for polycrystalline, nonepitaxial $\mathrm{Fe} / \mathrm{MgO} / \mathrm{Al}$ junctions [2], the tunneling conductance is more suppressed at lower bias and displays a larger peak near $V \sim \Delta$ expected from the BCS density of states.

While the absolute conductance could be inferred from the BTK model [30], such that in the absence of an interfacial barrierthe $Z=0$ case recovers the value of the Sharvin conductance [1], various deviations have been known for a while [34]. In $F / I / S$ junctions the conductance can be several orders of magnitude lower than predicted by the BTK model. However, the relevance of a BTK-like description is still ascertained by recognizing that it accurately describes the normalized-conductance spectra [34]. In our junctions reduced absolute conductance, as compared with (a)

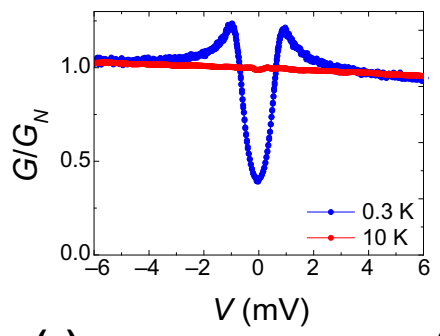

(c)

(b)

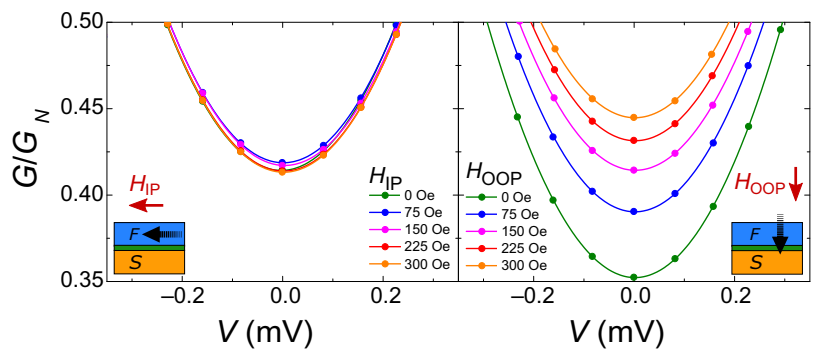

FIG. 3. Conductance dependence with bias and applied magnetic field, $H$. (a) Typical low-field conductance in $\mathrm{Fe} / \mathrm{MgO} / \mathrm{V}$ junctions at $H=0 \mathrm{Oe}$, below and above $T_{C}$. (b) Evolution of the zero-bias conductance with in-plane (IP) and out-of-plane (OOP) magnetic field at $T=0.3 \mathrm{~K}$. The two remanent perpendicularly oriented magnetic states (black arrows) with different $G(0)$ reveal approximately $17 \%$ conductance anisotropy (MAAR) at $H=0$. (c),(d) The low-bias dependence of the conductance at $T=0.3 \mathrm{~K}$ shows a different behavior with an in-plane magnetic field and an out-of-plane magnetic field. The conductance is normalized to its normal-state value, $G_{N}=G(V=3 \mathrm{mV})$.

the BTK predictions, can be inferred from Fig. 3(b). This is consistent with the epitaxial character of our $F / I / S$ junctions. Even if the interfacial barrier is removed, symmetry-enforced spin filtering would not a give a simple limit of Sharvin conductance, which ignores the relevant spin-related electronic structure and the role of SOC in spin filtering. In our control all-epitaxial $N / I / S$ sample $\mathrm{Au}(15 \mathrm{~nm}) / \mathrm{MgO}(2 \mathrm{~nm}) / \mathrm{V}(40 \mathrm{~nm})$, grown by the same method as the samples in Fig. 1, replacing Fe by Au leads to a 1000-fold larger conductance. This striking increase cannot be explained by the approximately- $0.2-\mathrm{eV}$ reduction in the interfacial barrier, but rather shows the importance of the symmetry-imposed spin filtering that is absent with Au.

To minimize $H$-dependent MAAR effects, potentially leading to anisotropy because of the vortex distribution in the superconductor, in Fig. 3(b) we identify the lower bound of MAAR by performing remanent measurements at $H=0$. While such a goal to realize multiple magnetization orientations, stable at $H=0$, is impossible in typical $F / I / S$ junctions, a unique feature in the design of our structures is their multiple nonvolatile states, depicted in Fig. 3(b), resulting from the competing perpendicular and in-plane anisotropy [29]. We first apply and then remove an in-plane saturation field of approximately $4 \mathrm{kOe}$ 
in the required direction. In the second stage, the field dependences of conductance are measured by initiating the field sweeps from those different remanent states. The corresponding measurements of $G(0)$ and $G(\pi / 2)$ at $V=0$ confirm a giant increase in the magnetic anisotropy of the superconducting state with a MAAR of approximately $17 \%$.

An applied magnetic field, $H$, leads to vortex formation and a gradual suppression of superconductivity in $V$, which is destroyed at a critical field $H=H_{C 2}$. For our thin V film, the measured $H_{C 2}$ is anisotropic: $H_{C 2, \perp}=3.5 \mathrm{kOe}$ and $H_{C 2, \|}=12 \mathrm{kOe}$. From a given range of $H$ in Figs. 3(c) and 3(d), we can also infer such $H_{C 2}$ anisotropy from lowbias $G(V)$. There is only a negligible $G(V)$ change for an in-plane magnetic field, since $H / H_{C 2, \|} \ll 1$. For an out-ofplane magnetic field we see a larger change; an increase of $G(V)$ is expected for suppression of superconductivity.

From the $H=0$ data in Figs. 3(b)-3(d) we can rule out that MAAR is a trivial effect due to the induced vortices with an applied magnetic field. The observed MAAR also substantially exceeds the possible effect of the fringing fields from the $\mathrm{Fe}$ region on $\mathrm{V}$ film separated by $\mathrm{MgO}$, as evaluated from micromagnetic simulations using the MuMax $^{3}$ code [39] (less than 20 Oe in the central region and less than 150 Oe near the edges, still less than $H_{C 1}$ for $\left.\mathrm{V}[40,41]\right)$. We verify on a control all-epitaxial $\mathrm{Au} / \mathrm{MgO} / \mathrm{V}$ junction that applying a perpendicular magnetic field in the amount of these calculated fringing fields changes the measured conductance by less than $1 \%$. If vortices at $H=0$ were formed by such fringing fields, their effect on $\mathrm{V}$ film would be stronger for an out-ofplane magnetization and thus show stronger suppression of superconductivity as reflected by a larger $G(V=0)$, which would be closer to the normal-state value, $G_{N}$. Instead, we see from Figs. 3(c) and 3(d) an opposite effect: $G(V=0)$ is smaller for an out-of-plane magnetization, which is inconsistent with a stronger suppression of superconductivity and MAAR dominated by vortices.

Remarkably, compared with Fig. 2, in Fig. 3 there is a huge increase in the observed anisotropy, which is seemingly inconsistent with only a very small interfacial SOC responsible for TAMR. To reconcile such an increase in magnetic anisotropy it is useful to recognize the sensitivity of Andreev reflection to interfacial SOC for a highly-spinpolarized ferromagnet [1]. In the limiting case of complete spin polarization with the absence of minority spins, no conventional Andreev reflection [Fig. 1(c)] is expected and thus $G(V<\Delta)=0$. However, with interfacial SOC, a spin-flip [equal spin, see Fig. 1(c)] Andreev reflection is possible, supporting the triplet superconductivity.

\section{MODEL AND DISCUSSION}

Orbital-symmetry-controlled tunneling turns $\mathrm{Fe} / \mathrm{MgO}$ into a source of highly-spin-polarized carriers [1-3], while

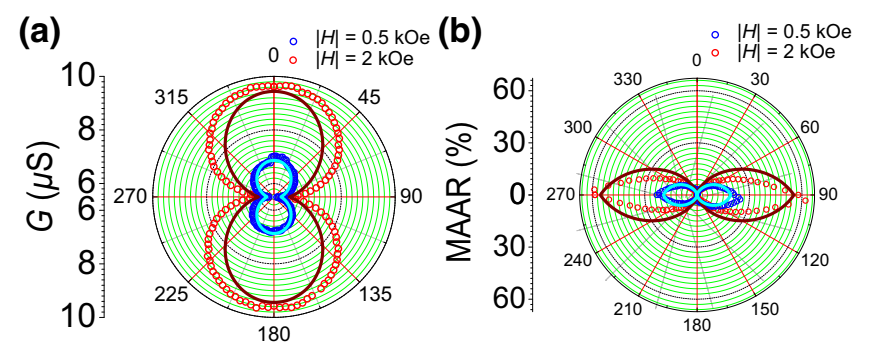

FIG. 4. MAAR. (a) Out-of-plane zero-bias conductance anisotropy at $T=0.3 \mathrm{~K}$ and $H=0.5 \mathrm{kOe}$ (blue dots) and $H=$ $2 \mathrm{kOe}$ (red dots) compared with our phenomenological model including magnetic field effects (solid lines) with fitting parameters (see the text) $G_{0}=6.01 \mu \mathrm{S}, A=-0.0415 \mu \mathrm{S}$, and $B=$ $-0.224 \mu \mathrm{S}$. (b) The same approach for out-of-plane MAAR.

the structural inversion asymmetry in the $\mathrm{Fe} / \mathrm{MgO} / \mathrm{V}$ junction leads to an interfacial Rashba SOC $[1,4]$ with the field $w_{R}=\left(\alpha k_{y},-\alpha k_{x}\right)$, where $\alpha$ is the interfacial-RashbaSOC parameter and $\mathbf{k}_{\|}=\left(k_{x}, k_{y}\right)$ is the in-plane wave vector. While the presence of interfacial SOC can be already inferred from $G(\theta)$ in Fig. 4(a), it is also helpful to examine the corresponding out-of-plane MAAR [22] from Eq. (1), shown in Fig. 4(b). Further support for the interfacial SOC comes from $G(0)$ in Fig. 3(a): its suppression expected for a highly-spin-polarized (approximetaly $70 \%$ ) $\mathrm{Fe} / \mathrm{MgO}$ interface [1] is rather small as compared with $G(V \gg \Delta)$, pointing to unconventional Andreev reflection as the main contribution to $G(0)$. For $V \gg \Delta$, shot-noise measurements (see Appendix B) confirm electron tunneling, excluding the presence of pinholes as the origin of a relatively high $G(0)$.

Although the measured MAAR and its relation to the interfacial SOC appear to be in qualitative agreement with previous theoretical predictions [22], sizable magnetic field effects result in a large discrepancy with respect to the magnitude and angular dependence of the MAAR (see Appendix D). In $F / I / S$ junctions the rotation of magnetization, in a finite magnetic field, generally yields two additional effects that were previously not considered [22]: orbital contributions and $H$-dependent suppression of superconductivity. The orbital effects are related to the Lorentz force and cyclotron orbits, which we include phenomenologically [4] by shifting the initial $\mathbf{k}_{\|}$in the reflection probability and the SOC field, resulting in the conductance (see Appendix D)

$$
G^{j}(V, \theta, H)=G_{0}^{j}+G_{\alpha}{ }^{j}(1-\cos 2 \theta)
$$

separated into a SOC-independent part $G_{0}{ }^{j}$ and a SOCdependent part $G_{\alpha}{ }^{j}$ part, with $G_{\alpha}{ }^{j}=g_{1}{ }^{j} \alpha H+g_{2}{ }^{j} \alpha^{2}$, where $g_{1}^{j}$ and $g_{2}^{j}$ are real and SOC-independent coefficients, while index $j=N, S$ labels the normal and superconducting contributions. The other effect of an applied 
magnetic field, as discussed above, is the vortex formation and a gradual suppression of superconductivity, which is destroyed at $H=H_{C 2}(\theta)$ [35]. From our measured values of $H_{C 2, \perp}$ and $H_{C 2, \|}$, the angular dependence of $H_{C}$ in a $\mathrm{V}$ thin film can be described by Tinkham's formula: $\left.\left[H_{C 2}(\theta) / H_{C 2, \|}\right)\right]^{2} \sin ^{2} \theta+\left[H_{C 2}(\theta) / H_{C 2, \perp}\right]|\cos \theta|=1$ (see Appendix D and Ref. [42]). We define a dimensionless field $h(\theta)=H / H_{C 2}(\theta)$ that quantifies the creation of vortices introducing a normal contribution to conductance, as well as the suppression of its superconducting part (see Ref. [35] and Appendix D),

$$
G(V, \theta, H)=h(\theta) G^{N}+[1-h(\theta)] G^{S} .
$$

In our analysis of the $G(V=0)$ data for a complete rotation of magnetization from Fig. 4(a), we apply the phenomenological model described by Eqs. (2) and (3), where we assume $G^{N}=2 G^{S}$, as in the measured $G(V=0)$ from Fig. 10(b) and omit the index $S$. From the $G(\theta)$ data at $H=0.5$ and $2 \mathrm{kOe}$, we first determine $G_{0}$ at $\theta=0$ and then solve for the two parameters $A=g_{1} \alpha H_{C, \perp}$ and $B=$ $g_{2} \alpha^{2}$ at $\theta=3 \pi / 2$. While we use the measured data for only these two angles, we recover good agreement in both $G$ and MAAR over the full range of $\theta$ shown in Figs. 4(a) and 4(b) with solid lines. This also yields an expected $G_{0} \gg G_{\alpha}$ from our model, corroborating the role of interfacial SOC in the giant MAAR of approximately $20 \%$ at $0.5 \mathrm{kOe}$ and approximately $60 \%$ at $2 \mathrm{kOe}$ [38]. The agreement between our phenomenological model and the data for $G(\theta)$ and $\operatorname{MAAR}(\theta)$ is even better for smaller $H=$ $0.5 \mathrm{kOe}$, since this model contains only the leading corrections to the applied magnetic field.

The nonvolatile control of magnetization and modifications of superconductivity can be extended in $F 1 / I / F 2 / I / S$ junctions, as shown in Fig. 5, to realize four different states: parallel, antiparallel, perpendicular in plane, and perpendicular out of plane. While similar spin-valve structures are common to superconducting spintronics [5-7], within our platform they reveal further opportunities due to the presence of additional nonvolatile configurations that are realized at $H=0$. Conductance variations above the gap in Fig. 5 resemble findings in $F / S$-based structures from Ref. [43], explained by long-range triplet, and show peculiar trends that could motivate further theoretical studies of interfacial SOC in all-epitaxial junctions. These observed conductance anomalies are periodic (see Appendix $\mathrm{C}$ showing typical conductance anomalies in a wider bias range, disappear slightly above $T_{C}$, and are sensitive to the relative magnetization orientation. They are likely to arise from quasiparticle interference inside 10-nm-thick Fe surrounded by two $\mathrm{MgO}$ layers, while the presence of the two periods above the gap was explained in Ref. [44] as a result of shorter and longer coherent electron-hole trajectories. Different magnitudes in conductance variations for two perpendicular configurations,

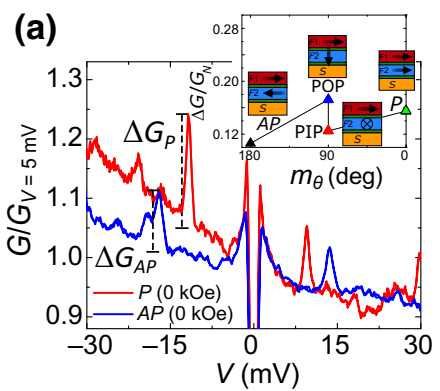

(b)

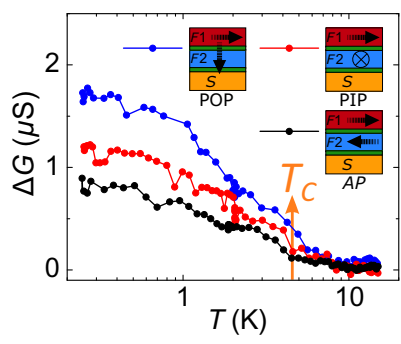

FIG. 5. Conductance characterization of $F 1 / I / F 2 / I / S$ junctions at remanence. (a) Bias dependence of the conductance normalized by its above-the-gap value at $V=5 \mathrm{mV}$ and $T=0.3 \mathrm{~K}$ for parallel (P) and antiparallel (AP) magnetization orientation (black arrows). $\Delta G$ denotes the above-the-gap largest conductance anomalies. The inset shows the variation of the normalized largest $\Delta G$ (observed around $-15 \mathrm{mV}$ ) with the magnetization orientation parallel, antiparallel, perpendicular out of plane (POP), and perpendicular in plane (PIP). The top $F$ region is magnetically hard $(\mathrm{Fe} / \mathrm{Co})$ and the bottom $F$ region $(\mathrm{Fe})$ is soft. (b) The temperature dependence of the amplitude of the strongest conductance anomaly for different magnetic states: POP, PIP, and AP.

with magnetization in $F 2$ being in plane versus out-ofplane, suggest unconventional pairing. Unlike the expected weakest suppression of spin-singlet superconductivity with antiparallel magnetization [10], the amplitude of conductance anomalies in the antiparallel configuration is suppressed for all $T<T_{C}$ as compared with the other two magnetization orientations. These variations remain practically unchanged for normalization at $-5 \mathrm{mV}$ and increase if the absolute values are compared. Since the changes in the normalized conductance below $T_{C}$ can reach up to $50 \%$ (between antiparallel and perpendicular out of plane, Fig. 5 inset), multiple ferromagnet regions can support previously unexplored MR effects. Another extension of our all-epitaxial junctions would be to include the second superconducting layer and explore the modification of the Josephson effect from the interplay between the interfacial SOC and different magnetic configurations.

\section{CONCLUSIONS}

Our platform of all-epitaxial $F / I / S$ junctions could both stimulate theoretical studies beyond the BTK-like description to include symmetry-controlled spin filtering and enable experimental progress toward applications in superconducting spintronics. A common requirement to realize SOC-driven emergent phenomena, from topological states to long-range triplet, is typically SOC, which is already inherently strong in the normal state, for example, as implemented with heavy elements and in narrow-band semiconductors $[16,45,46]$. In contrast, the platform we study reveals a peculiar superconducting behavior even when a rather weak SOC in the normal state leads to 
negligible magnetic anisotropy. Further experiments with this platform could also test a recent prediction about the $\pi / 4$ change of the in-plane magnetic easy axis below $T_{C}$ [47] as further support for the efficient generation of spin-triplet Cooper pairs, excluding alternative scenarios where our observed conductance changes with magnetization direction would be attributed to trapped vortices or manifestations of quasiparticle transport. We expect that revisiting $\mathrm{Fe} / \mathrm{MgO}$-based junctions, which are widely used in commercial spintronic applications, will provide an opportunity to use multiple proximity effects - spin-orbit, magnetic, and superconducting - to transform a large class of materials and realize unexplored phenomena [24].

\section{ACKNOWLEDGMENTS}

We thank Tong Zhou and Diego Caso for help with micromagnetic simulations. The work in Madrid was supported in part by Spanish Ministerio de Ciencia, Innovación y Universidades (Grants No. MAT2015-66000P, No. RTI2018-095303-B-C55, No. EUIN2017-87474, and No. MDM-2014-0377) and Comunidad de Madrid (Grant No. NANOMAGCOST-CM P2018/NMT-4321). C.T. acknowledges EMERSPIN Grants No. PN-III-P4ID-PCE-2016-0143 and No. UEFISCDI:22/12.07.2017. J.P.C. acknowledges support from a Fundación Séneca (Región de Murcia) postdoctoral fellowship (Grant No. 19791/PD/15). The work in Regensburg was supported by the Deutsche Forschungsgemeinschaft (DFG, German Research Foundation) - Project-ID314695032 - SFB 1277, International Doctorate Program Topological Insulators of the Elite Network of Bavaria, and GRK 1570. The work in Nancy was supported by CPER MatDS and the French PIA project "Lorraine Université d'Excellence," reference ANR-15-IDEX-04-LUE. Experiments were performed using equipment from the TUBE [48]. DAUM [48] was funded by FEDER (EU), ANR, Région Grand Est, and Metropole Grand Nancy. The work in Detroit was supported by the U.S. Office of Naval Research (Grant No. N000141712793) and Defense Advanced Research Projects Agency (Grant No. DP18AP900007). The work in Buffalo was supported by the U.S. Department of Energy, Basic Energy Sciences (Grant No. DE-SC0004890).

I.M., P.H., and C.G.-R. contributed equally to the manuscript.

\section{APPENDIX A: COMPARISON BETWEEN TAMR AND MAAR}

TAMR and MAAR provide important information about the anisotropy of the transport properties in the normal state and the superconducting state, respectively (see the main text). They have the same functional form that can be expressed in terms of the angle-dependent conductance in the normal state and the superconducting state [22]:

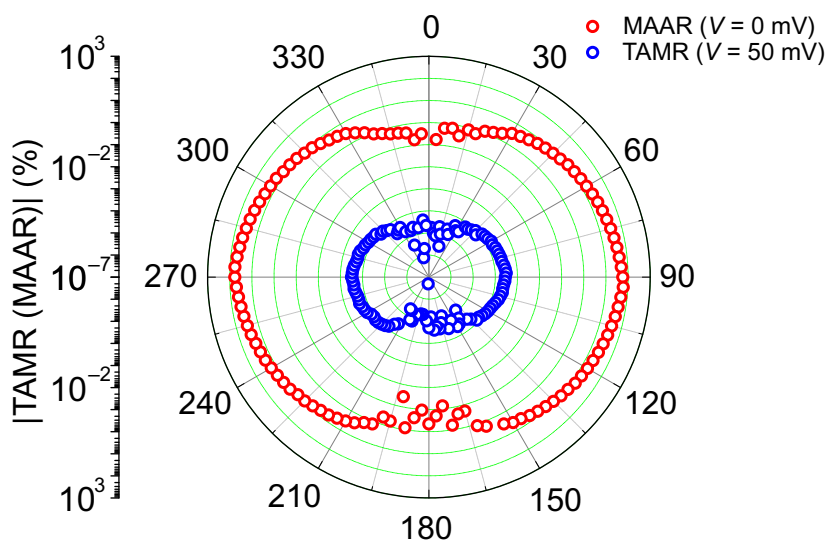

FIG. 6. Comparison between TAMR and MAAR. Measurements are performed at $T=0.3 \mathrm{~K}$ and an applied bias $V=$ $50 \mathrm{mV}$ (TAMR) and $V=0 \mathrm{mV}$ (MAAR) with applied magnetic field $H=2 \mathrm{kOe}$. The vanishing TAMR and MAAR for the angle $\theta=0^{\circ}$ are excluded to avoid a diverging logarithm.

$$
\operatorname{TAMR}(\operatorname{MAAR})=\frac{G(0)-G(\theta)}{G(\theta)},
$$

where the angle $\theta$ is measured between the magnetization, $\mathbf{M}$, and the interface normal of the junction, such that an out-of-plane rotation of magnetization is considered.

To provide a direct comparison of TAMR and MAAR, which for the same sample have amplitudes that are several orders of magnitude different, we show their logarithmic plots and exclude the $\theta=0^{\circ}$ results (to avoid a diverging logarithm). The corresponding results are shown in Fig. 6 for an all-epitaxial $F / I / S \mathrm{Fe} / \mathrm{MgO} / \mathrm{V}$ junction as depicted in Fig. 1. The distinction between the normal state and the superconducting state, and thus between the TAMR and the MAAR, is realized by changing the applied bias, $V$-above the superconducting gap of vanadium approximately $1 \mathrm{meV}$ or less $(V \gg \Delta)$ for TAMR and $V=0$ for MAAR - while the sample temperature $T=0.3 \mathrm{~K}$ and applied magnetic field $H=2 \mathrm{kOe}$ are kept fixed.

\section{APPENDIX B: SHOT-NOISE CHARACTERIZATION OF BARRIER QUALITY}

Shot noise is an intrinsic quantum property arising from the discreteness of the charge carriers [49]. It is convenient to express its dimensionless form as a Fano factor, noiseto-current ratio, since it can attain universal values that are independent of the details of the system [23,25]. Such a Fano factor is used to characterize MTJs and the corresponding TMR since it depends on the relative orientation of the magnetization in different ferromagnets [25]. For example, for MTJs with a single barrier region, the Fano factor approaches 1 in the tunneling limit [23]. The observation of such a value in Fig. 7 indicates direct tunneling through a pinhole-free barrier. With two possible magnetic states in double-barrier $F / I / F / I / S$ junctions, the expected 


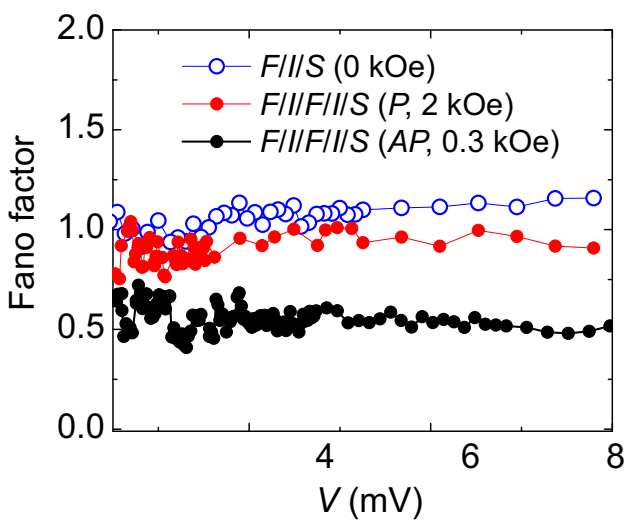

FIG. 7. Bias dependence of the Fano factor for $F / I / S$ and $F / I / F / I / S$ junctions in the parallel $(\mathrm{P})$ state $(H=2 \mathrm{kOe})$ and antiparallel (AP) state $(H=0.3 \mathrm{kOe})$ measured at $T=0.3 \mathrm{~K}$ for bias exceeding the superconducting gap $(\Delta \lesssim 1 \mathrm{meV})$.

shot noise is evaluated with use of a model of sequential tunneling $[23,50]$ The influence of resonant tunneling is not included. The measured Fano factor for the parallel and antiparallel magnetic configurations can be used to calculate the TMR ratio. The corresponding value shows good agreement with the measured TMR (in the limit of strong spin relaxation).

Following the calculation of shot noise in the presence of spin relaxation [50], the Fano factor is given by

$$
F=\frac{R_{2 \uparrow}^{2} R_{2 \downarrow}^{2}\left(R_{1 \uparrow}+R_{1 \downarrow}\right)^{2}+R_{1 \uparrow}^{2} R_{1 \downarrow}^{2}\left(R_{2 \uparrow}+R_{2 \downarrow}\right)^{2}}{\left[R_{1 \uparrow} R_{1 \downarrow}\left(R_{2 \uparrow}+R_{2 \downarrow}\right)+R_{2 \uparrow} R_{2 \downarrow}\left(R_{1 \uparrow}+R_{1 \downarrow}\right)\right]^{2}},
$$

where $R$ is the partial resistance of each of the two barriers (indices 1 and 2) and of each of the spin directions (up $\uparrow$ and down $\downarrow$ ).

For the $F / I / F / I / S$ junction the two barriers separate different systems. The normal-state resistance of the $F / I / S$ system (for $V>\Delta$ ), $R_{F I S}$, is largely independent of the relative magnetic configuration of the two ferromagnet electrodes. This is in contrast to the $F / I / F$ system, which has different resistances for parallel and antiparallel configurations, $R_{F / I / F}^{P, A P}$. By defining $\alpha_{P, A P}=R_{F / I / F}^{P, A P} / R_{F / I / S}$, we can express the total resistance of the $F / I / F / I / S$ system as

$$
R_{T}=R_{F / I / F}+R_{F / I / S}=\left(\alpha_{P, A P}+1\right) R_{F / I / S} .
$$

With this notation, the Fano factor is given by

$$
F_{P, A P}=\left(1+\alpha_{P, A P}^{2}\right) /\left(1+\alpha_{P, A P}\right)^{2},
$$

while the expression for TMR is

$$
\operatorname{TMR}=\left(\alpha_{A P}-\alpha_{P}\right) /\left(1+\alpha_{P}\right) .
$$

By using the measured Fano factors in Fig. 7 averaged over bias with $F_{P}=0.94 \pm 0.1$ and $F_{A P}=0.58 \pm 0.1$, we obtain $F / I / F / I / S$ TMR of about $40 \%$, which is consistent with the TMR measured from the corresponding parallel and antiparallel conductance, thus further corroborating tunneling without pinholes in our junctions.

\section{APPENDIX C: PERIODICITY OF ABOVE-THE-GAP CONDUCTANCE ANOMALIES}

In this section we present the conductance anomalies observed above the gap on $F 1 / I / F 2 / I / S$ junctions, shown in Fig. 5, for a broader bias range (Fig. 8). The results are similar to those found in Ref. [43]. The apparent lack of periodicity in Fig. 5 is due to the limited bias range selected to show how the amplitude of one specific conductance anomaly varies with the four possible magnetization alignments.

\section{APPENDIX D: $F / I / S$ MODEL IN THE PRESENCE OF SPIN-ORBIT COUPLING AND AN EXTERNAL MAGNETIC FIELD}

To model the $F / I / S$ junction with interfacial SOC, where the $F(S)$ region is semiinfinite at $z<0(z>0)$, we use a generalized BTK formalism [22] and solve the Bogoliubov-de Gennes equation [51] for quasiparticle states $\Psi(\mathbf{r})$ with energy $E$ :

$$
\left(\begin{array}{cc}
\hat{H}_{e} & \hat{\Delta} \\
\hat{\Delta}^{\dagger} & \hat{H}_{h}
\end{array}\right) \Psi(\mathbf{r})=E \Psi(\mathbf{r})
$$
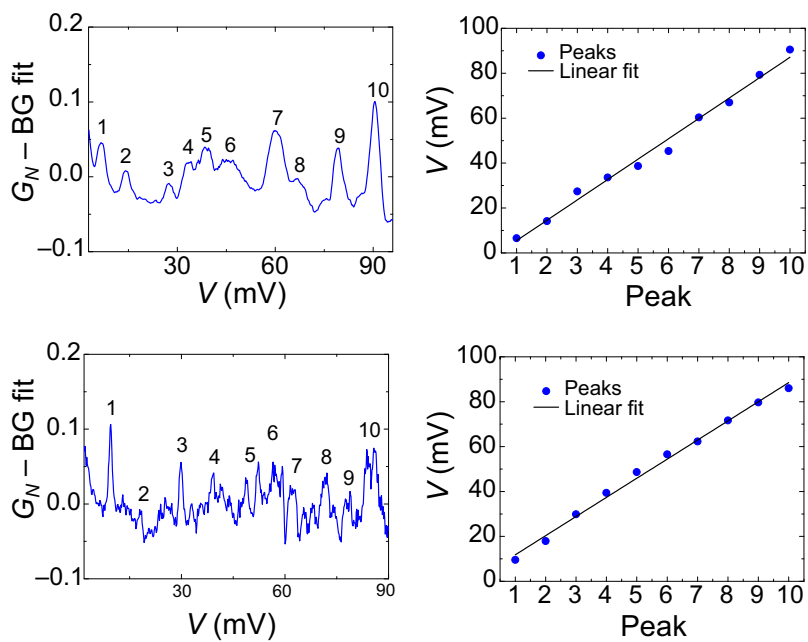

FIG. 8. Periodicity of the above-the-gap conductance anomalies for two different $F 1 / I / F 2 / I / S$ junctions in the parallel state at $0.3 \mathrm{~K}$. Left: normalized $I-V$ curves from above the gap to $V \sim 90 \mathrm{mV}$, where a background fit (BG fit) has been subtracted from each $G_{N}(V)$ curve. The selected conductance anomalies (peaks) are marked with numbers. Right: peak number versus bias for the two $G_{N}(V)$ curves, with a linear fit that shows how they fit to a periodic pattern. The lower panels are obtained from the same $G_{N}(V)$ curve shown in Fig. 5(a). Only the bias positions of the clearest peaks are counted. 
where the single-particle Hamiltonian for electrons is $\hat{H}_{e}=-\left(\hbar^{2} / 2\right) \nabla[1 / m(z)] \nabla-\mu(z)-\left(\Delta_{x c} / 2\right) \theta(-z) \mathbf{m} \cdot \hat{\boldsymbol{\sigma}}$ $+\left(V_{0} d+\mathbf{w} \cdot \hat{\boldsymbol{\sigma}}\right) \delta(z)$ and for holes is $\hat{H}_{h}=-\hat{\sigma}_{y} \hat{H}_{e}^{*} \hat{\sigma}_{y}$. They contain the effective mass $m(z)$, the chemical potential $\mu(z)$, and the exchange spin splitting $\Delta_{x c}$. The unit magnetization vector in the $x-z$ plane is $\mathbf{m}=$ $(\sin \theta, 0, \cos \theta)$ and $\hat{\sigma}$ are Pauli matrices. The $s$-wave superconductor $\mathrm{V}$ is modeled by the pair potential $\Delta \theta(z) \mathbb{1}_{2 \times 2}$ with the isotropic gap $\Delta$. At $z=0$ we assume a flat interface, at which we account for the insulating $\mathrm{MgO}$ layer by including a $\delta$-like potential barrier $V_{0} d \delta(z)$, with effective height $V_{0}$ and width $d$. Since the built-in electric field due to structural inversion asymmetry inducing Rashba SOC is largest at interfaces, the Rashba SOC field $[1,4]$

$$
\mathbf{w}_{R}=\left(\alpha k_{y},-\alpha k_{x}\right)
$$

is also considered to be $\delta$-like. Because of the conservation of in-plane wave vector $\mathbf{k}_{\|}$, we can write $\Psi_{\sigma}(\mathbf{r})=$ $\Psi_{\sigma}(z) e^{i \mathbf{k}_{\|} \mathbf{r}_{\|}}$. We find the solution in the ferromagnet layer for incoming electrons with spin $\sigma$

$$
\begin{aligned}
\Psi_{\sigma}^{F}= & \frac{1}{\sqrt{k_{\sigma}^{e}}} e^{i k_{\sigma}^{e} z} \chi_{\sigma}^{e}+r_{\sigma, \sigma}^{e} e^{-i k_{\sigma}^{e} z} \chi_{\sigma}^{e}+r_{\sigma,-\sigma}^{e} e^{-i k_{-\sigma}^{e} z} \chi_{-\sigma}^{e} \\
& +r_{\sigma,-\sigma}^{h} e^{i k_{-\sigma}^{h} z} \chi_{-\sigma}^{h}+r_{\sigma, \sigma}^{h} e^{i k_{\sigma}^{h} z} \chi_{\sigma}^{h},
\end{aligned}
$$

with electronlike $\chi_{\sigma}^{e}=\left(\chi_{\sigma}, 0\right)^{T}$ and holelike $\chi_{\sigma}^{h}=$ $\left(0, \chi_{-\sigma}\right)^{T}$ spinors, where

$$
\chi_{\sigma}^{T}=(\sigma \sqrt{1+\sigma \cos \theta}, \sqrt{1-\sigma \cos \theta}) / \sqrt{2}
$$

and $\sigma=1(-1)$ corresponds to the spin parallel (antiparallel) to $\hat{\mathbf{m}}$. The scattering coefficients are specular reflection $r_{\sigma, \sigma}^{e}$, specular reflection with spin flip $r_{\sigma,-\sigma}^{e}$, conventional Andreev reflection $r_{\sigma,-\sigma}^{h}$, and Andreev reflection with spin flip $r_{\sigma, \sigma}^{h}$. The electronlike (holelike) wave vectors in the ferromagnet region are $k_{\sigma}^{e(h)}=\sqrt{k_{F}^{2}+2 m_{F} / \hbar^{2}\left[(-) E+\sigma \Delta_{x c} / 2\right]-k_{\|}^{2}}$. The superconducting scattering states are

$$
\begin{aligned}
\Psi_{\sigma}^{S}= & t_{\sigma, \sigma}^{e} e^{i q^{e} z}\left(\begin{array}{l}
u \\
0 \\
v \\
0
\end{array}\right)+t_{\sigma,-\sigma}^{e} e^{i q^{e} z}\left(\begin{array}{l}
0 \\
u \\
0 \\
v
\end{array}\right) \\
& +t_{\sigma, \sigma}^{h} e^{-i q^{h} z}\left(\begin{array}{l}
v \\
0 \\
u \\
0
\end{array}\right)+t_{\sigma,-\sigma}^{h} e^{-i q^{h z}}\left(\begin{array}{l}
0 \\
v \\
0 \\
u
\end{array}\right),
\end{aligned}
$$

with superconducting coherence factors $u^{2}=1-v^{2}=$ $\left(1+\sqrt{E^{2}-\Delta^{2}} /|E|\right) / 2$. The states comprise scattering coefficients for electronlike (holelike) transmission without spin flip $t_{\sigma, \sigma}^{e}\left(t_{\sigma, \sigma}^{h}\right)$ and electronlike (holelike) transmission with spin flip $t_{\sigma,-\sigma}^{e}\left(t_{\sigma,-\sigma}^{h}\right)$. The wave vectors are given by $q^{e(h)}=\sqrt{q_{F}^{2}+(-) 2 m_{S} / \hbar^{2} \sqrt{E^{2}-\Delta^{2}}-k_{\|}^{2}}$. Applying charge-current conservation, we compute the differential conductance at zero temperature:

$$
G(V, \theta)=\frac{e^{2} A}{(2 \pi)^{2} h} \sum_{\sigma} \int d^{2} \mathbf{k}_{\|}\left[1+R_{\sigma}^{h}(-e V)-R_{\sigma}^{e}(e V)\right] .
$$

Here the probability amplitudes in the ferromagnet region, $R_{\sigma}^{e(h)}\left(E, \mathbf{k}_{\|}\right)=\operatorname{Re}\left(k_{\sigma}^{e(h)}\left|r_{\sigma, \sigma}^{e(h)}\right|^{2}+k_{-\sigma}^{e(h)}\left|r_{\sigma,-\sigma}^{e(h)}\right|^{2}\right)$, contain the scattering coefficients for specular and Andreev reflection with and without spin flip, $A$ is the interfacial area, and we use Andreev approximation $k_{\sigma}^{e}=k_{\sigma}^{h}$ [30].

To describe the $\mathrm{Fe} / \mathrm{MgO} / \mathrm{V}$ junction we use the Fermi wave vectors $k_{F}=q_{F}=0.805 \times 10^{8} \mathrm{~cm}^{-1}$ for $\mathrm{Fe}$ and $\mathrm{V}$, respectively. The effective masses are $m_{\mathrm{Fe}}=m_{\mathrm{V}}=m_{0}$, where $m_{0}$ is the free-electron mass. The spin polarization of $\mathrm{Fe}$ is given by $P=\left(\Delta_{x c} / 2\right) / \mu_{\mathrm{Fe}}=0.7$, where $\mu_{\mathrm{Fe}}=\hbar^{2} k_{F}^{2} /\left(2 m_{\mathrm{Fe}}\right)$. The gap for $\mathrm{V}$ is $\Delta=0.8 \mathrm{meV}$. The choice of the effective width and height of the epitaxially grown $\mathrm{MgO}$ insulating barrier, $d=1.7 \mathrm{~nm}$ and $V_{0}$, is guided by our low-bias experimental measurements. The SOC parameter $\alpha$ is adjusted to fit the experimental results. The parameters are further discussed in Appendix E. From the boundary conditions ensuring probability conservation

$$
\begin{gathered}
\left.\Psi_{\sigma}^{F}\right|_{z=0^{-}}=\left.\Psi_{\sigma}^{S}\right|_{z=0^{+}}, \\
\left.\frac{\hbar^{2}}{2 m_{S}} \frac{d}{d z} \eta \Psi_{\sigma}^{S}\right|_{z=0^{+}}=\left.\left(\begin{array}{cc}
\mathbf{w} \cdot \hat{\boldsymbol{\sigma}} & 0 \\
0 & -\mathbf{w} \cdot \hat{\boldsymbol{\sigma}}
\end{array}\right) \Psi_{\sigma}^{F}\right|_{z=0^{-}} \\
+\left.\left(\frac{\hbar^{2}}{2 m_{F}} \frac{d}{d z}+V_{0} d\right) \eta \Psi_{\sigma}^{F}\right|_{z=0^{-}}
\end{gathered}
$$

with

$$
\eta=\left(\begin{array}{cc}
\mathbb{1}_{2 \times 2} & 0 \\
0 & -\mathbb{1}_{2 \times 2}
\end{array}\right),
$$

scattering coefficients are obtained numerically to give the resulting conductance by our performing the integration in Eq. (D6). For a low-bias $|e V|<\Delta$ quasiparticle transmission is prohibited and we get from probability current conservation $R_{\sigma}^{e}(\mathrm{eV})=1-R_{\sigma}^{h}(\mathrm{eV})$, which leads to

$$
G(V, \theta)=\frac{e^{2} A}{(2 \pi)^{2} h} \sum_{\sigma} \int d^{2} \mathbf{k}_{\|}\left[2 R_{\sigma}^{h}(e V)\right] .
$$

Thus, zero-bias conductance depends only on the probability amplitude of Andreev reflection. 
The calculated angular dependences of $G$ and MAAR are presented in Fig. 9. To get more insight into the physical mechanisms behind the angular dependence of $G$ and MAAR, we consider a simple phenomenological model that was developed earlier for TAMR [4,52] and also applied to MAAR [22]. The model is based on general symmetry arguments. It identifies two preferential directions in the system for given $\mathbf{k}_{\|}$: namely, $\mathbf{m}$ and $\mathbf{w}\left(\mathbf{k}_{\|}\right)$. Therefore, a scalar quantity as the Andreev reflection probability can be expanded in powers of $\mathbf{m} \cdot \mathbf{w}\left(\mathbf{k}_{\|}\right)$. Up to second order in SOC, we get for the conductance

$$
\begin{aligned}
G(V, \theta)= & \frac{e^{2} A}{(2 \pi)^{2} h} \sum_{\sigma} \int d^{2} \mathbf{k}_{\|} 2\left\{R_{\sigma}^{h,(0)}(e V)+R_{\sigma}^{h,(1)}(e V)\right. \\
& \left.\times\left[\mathbf{m} \cdot \mathbf{w}\left(\mathbf{k}_{\|}\right)\right]+R_{\sigma}^{h,(2)}(e V)\left[\mathbf{m} \cdot \mathbf{w}\left(\mathbf{k}_{\|}\right)\right]^{2}\right\} .
\end{aligned}
$$

The linear term vanishes after integration due to $\mathbf{w}\left(\mathbf{k}_{\|}\right)=$ $-\mathbf{w}\left(-\mathbf{k}_{\|}\right)$, and with Eq. (D2) we obtain

$$
G(V, \theta)=G_{0}+g_{2} \alpha^{2}(1-\cos 2 \theta),
$$

which contains the SOC-independent conductance $G_{0}=$ $e^{2} A /(2 \pi)^{2} / h \sum_{\sigma} \int d^{2} \mathbf{k}_{\|} 2 R_{\sigma}^{h,(0)}(e V)$ and the SOC-dependent part with the expansion coefficient $g_{2}=e^{2} A /(2 \pi)^{2} /$ $h \sum_{\sigma} \int d^{2} \mathbf{k}_{\|} R_{\sigma}^{h,(2)}(e V) k_{y}^{2}$. For the MAAR we get

$$
\operatorname{MAAR}(\theta)=\frac{g_{2} \alpha^{2}(\cos 2 \theta-1)}{G_{0}-g_{2} \alpha^{2}(\cos 2 \theta-1)} .
$$

To obtain the theoretical MAAR $(\theta)$ we choose the SOC value to match the experimental value at $\theta=270^{\circ}$; by definition $\operatorname{MAAR}\left(0^{\circ}\right)=0$. In Fig. $9($ b $)$ we observe a clear discrepancy between this $\operatorname{MAAR}(\theta)$ fit (black line) and experimental results at $H=0.5 \mathrm{kOe}$ (blue circles). The corresponding theoretical $G$ in Fig. 9(a) (black line), which was not fit, yields even weaker agreement. At higher fields for $H=2 \mathrm{kOe}$, as can be seen in Fig. 9(c), the deviation from the measured $\operatorname{MAAR}(\theta)$ is more pronounced. Since the MAAR amplitude grows with increasing $H$, we need two different values of the SOC parameter to match the magnitude in Figs. 9(b) and 9(c).

To take into account the influence of external magnetic field, which is required to rotate the magnetization in the $x-z$ plane, we recognize that there are two related effects: (i) orbital effects on the charge carriers and (ii) the suppression of superconductivity due to creation of vortices.

We first discuss orbital effects. We assume that the radius of the cyclotron orbits, on which charge carriers are forced by a magnetic field perpendicular to their propagation direction, is much larger than the width of the $\mathrm{MgO}$ tunnel barrier so that we can ignore effects from the external magnetic field. Since the influence of orbital effects from the external magnetic field is expected to be small, we include the magnetic field perturbatively in the phenomenological model for out-of-plane rotation of the magnetic field in a similar way as was proposed for the in-plane field [53]. A magnetic field is introduced into the model Hamiltonian using minimal coupling $\mathbf{p}=-i \hbar \boldsymbol{\nabla} \rightarrow \boldsymbol{\pi}=-i \hbar \boldsymbol{\nabla}+e \mathbf{A}$. The magnetic flux density is given by $\mathbf{B}=B \mathbf{m}$, so we can choose the gauge $\mathbf{A}=(-y B \cos \theta,-z B \sin \theta, 0)$ for the vector potential $\mathbf{A}$ and relate it to the magnetic field by $\mathbf{B}=\mu_{0} \mathbf{H}$, where $\mu_{0}$ is the vacuum permeability. With the substitution for the momentum above, the kinetic energy and Rashba SOC in the single-particle Hamiltonian $\hat{H}_{e}$ are modified as $\hat{H}_{\text {kin }}=1 / 2 \pi[1 / m(z)] \pi$ and $\hat{H}_{R}=\alpha / \hbar\left[\left(\pi_{y},-\pi_{x}, 0\right)\right.$. $\hat{\sigma}] \delta(z)$, respectively. Instead of numerically solving this problem, we want to study the underlying physical behavior. Our strategy is to again expand the Andreev reflection probability in powers of magnetization direction and SOC field. This is still possible, however, the expansion coefficients and SOC field are now $H$ dependent. The field-dependent quantities $R_{\sigma, H}^{h,(n)}(\mathrm{eV})$, where $n=0,1,2$ and $\mathbf{w}_{H}\left(\mathbf{k}_{\|}\right)$are approximated by the previous independent ones valid up to linear order in $H$. We first look at the
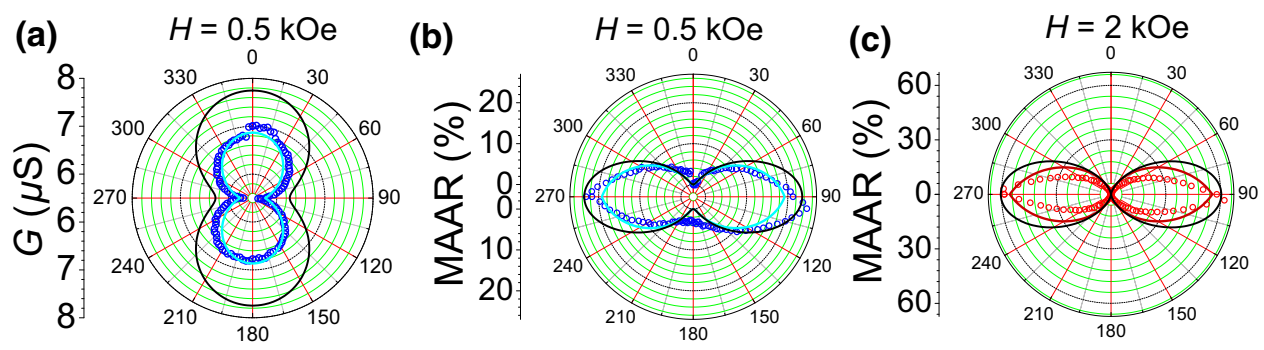

FIG. 9. (a) Out-of-plane $G(V=0, \theta)$ anisotropy measured at $T=0.3 \mathrm{~K}$ and $H=0.5 \mathrm{kOe}$ (blue dots) compared with (i) fits from the extended BTK model with $H=0, V_{0}=0.3 \mathrm{eV}$, and $\alpha=3.0 \mathrm{eV}^{2}$ (black line) and (ii) the phenomenological model including magnetic field effects with $G_{0}=6.01 \mu \mathrm{S}, A=-0.0415 \mu \mathrm{S}$, and $B=-0.224 \mu \mathrm{S}$ (blue line). (b) The same for the of out-of-plane $\operatorname{MAAR}(\theta)$ at $H=0.5 \mathrm{kOe}$. (c) Out-of-plane MAAR measured at $T=0.3 \mathrm{~K}$ and $H=2 \mathrm{kOe}$ (red dots) compared with (i) fits from the extended BTK model with $H=0, V_{0}=0.3 \mathrm{eV}$, and $\alpha=5.5 \mathrm{eV} \AA^{2}$ (black line) and (ii) the phenomenological model including magnetic field effects with $G_{0}, A$, and $B$ as specified above (red line). 
kinetic energy term. When $H=0$, the Andreev reflection is largest at $\mathbf{k}_{\|}=0$ since for finite $\mathbf{k}_{\|}$a part of the total kinetic energy of an incoming electron is in the direction parallel to the interface, which effectively increases the barrier height. When $H \neq 0$, the maximum Andreev reflection is shifted to an in-plane wave vector $\mathbf{k}_{\|, 0}$ which fulfills $\left\langle\left[k_{x, 0}-e \mu_{0} H y / \hbar \cos \theta\right]^{2}\right\rangle=0$ and $\left\langle\left[k_{y, 0}-e \mu_{0} H z / \hbar \sin \theta\right]^{2}\right\rangle=0$, performing a quantummechanical average $\langle\cdots\rangle$. Thus, the electrons feel effectively the smallest barrier for $\mathbf{k}_{\|, 0}=\left[b_{1} H \cos \theta, b_{2} H \sin \theta\right.$, $0]$, where $b_{1}$ and $b_{2}$ are constants that depend on $\langle y\rangle$ and $\left\langle y^{2}\right\rangle$ or $\langle z\rangle$ and $\left\langle z^{2}\right\rangle$, respectively. Thus, we approximate $R_{\sigma, H}^{h,(n)}\left(\mathbf{k}_{\|}\right) \approx R_{\sigma}^{h,(n)}\left(\sqrt{\left(k_{x}-k_{x, 0}\right)^{2}+\left(k_{y}-k_{y, 0}\right)^{2}}\right)$. This shift can be related to the Lorentz force, which sends the charge carriers on helicoids depending on the orientation of magnetization. Higher-order magnetic field effects on the Andreev reflection amplitude are ignored. The spinorbit field experiences also a momentum shift $\mathbf{w}_{H}\left(\mathbf{k}_{\|}\right) \approx$ $\mathbf{w}\left(k_{x}-b_{3} H \cos \theta, k_{y}-b_{4} H \sin \theta\right)$, where the coefficients $b_{3}$ and $b_{4}$, respectively, depend on $\langle y\rangle$ and $\langle z\rangle$ because momentum appears linearly in the SOC field.

In the presence of an out-of-plane magnetic field, the Hamiltonian is no longer translationally invariant in the $y$ direction. We assume that we can treat the spin-orbit and cyclotron effects as small perturbations and compute the conductance from $G(V, \theta)=$ $e^{2} A /(2 \pi)^{2} / h \sum_{\sigma} \int d^{2} \mathbf{k}_{\|} 2 R_{\sigma}^{h}(\mathrm{eV})$ by using the expansion of the Andreev reflection probability in powers of SOC with $H$-dependent expansion coefficients. The SOCindependent term is the same after integration as when $H=0$. Conductance corrections to the second-order term are ignored since they are quadratic in $H$. From the firstorder term, which vanishes for $H=0$, we get an additional contribution to the conductance due to the interplay of SOC and the magnetic field:

$$
\begin{aligned}
G_{\text {SOC-H }} & =\frac{e^{2} A}{(2 \pi)^{2} h} \sum_{\sigma} \int d^{2} \mathbf{k}_{\|} 2 R_{\sigma, H}^{h,(1)}(e V)\left[\mathbf{m} \cdot \mathbf{w}\left(\mathbf{k}_{\|}\right)\right] \\
& =g_{1} \alpha H(1-\cos 2 \theta),
\end{aligned}
$$

which is linear in $H$ and contains the coefficient $g_{1}=$ $e^{2} A /(2 \pi)^{2} / h \sum_{\sigma} \int d^{2} \mathbf{k}_{\|}^{\prime} 2 R_{\sigma}^{h,(1)}\left(k_{\|}^{\prime}\right)\left(b_{2}-b_{4}\right)$, with $\mathbf{k}_{\|}^{\prime}=$ $\mathbf{k}_{\|}-\mathbf{k}_{\|, 0}$. So orbital effects in the presence of a Rashba SOC field induce an angular dependence that is of the same form as the one from the second-order SOC term caused by the interplay of SOC and magnetization without considering the external magnetic field. The purely orbital contribution to the conductance, which is present for an out-of-plane magnetic field even without SOC, is ignored because it is of higher order in $H$. The conductance, which depends also on the magnetic field, is given by

$$
\begin{aligned}
G(V, \theta, H) & =G_{0}+\left(g_{1} \alpha H+g_{2} \alpha^{2}\right)(1-\cos 2 \theta) \\
& =G_{0}+G_{\alpha}(1-\cos 2 \theta) .
\end{aligned}
$$

Considering only this magnetic field correction is not enough since it does not cover the $G\left(\theta=0^{\circ}\right)$ increase [see Fig. 4(a)] and the modified shape of the angular dependence.

We next consider suppression of superconductivity in $\mathrm{V}$ as a type-II superconductor due to vortex formation with a magnetic field. In our thin superconducting films, the measured critical field for complete suppression of superconductivity is anisotropic: $H_{C 2, \perp}=3.5 \mathrm{kOe}\left(H_{C 2, \|}=\right.$ $12 \mathrm{kOe}$ ) when the magnetic field is applied perpendicular (parallel) to the film. The angular dependence of the critical field can be described by Tinkham's formula [42]:

$$
\frac{H_{C 2}^{2}(\theta)}{H_{C 2, \|}^{2}} \sin ^{2} \theta+\frac{H_{C 2}(\theta)}{H_{C 2, \perp}}|\cos \theta|=1 .
$$

We model the suppression of the superconductivity and the amount of normal conduction that is introduced by vortices by the ratio $h(\theta)=H / H_{C 2}(\theta)$. We assume a linear increase (decrease) of the normal (superconducting) conductance contribution with magnetic field [35]. The conductance becomes

$$
\begin{aligned}
G(V, \theta, H)= & h(\theta) G^{N}(V, \theta, H) \\
& +[1-h(\theta)] G^{S}(V, \theta, H),
\end{aligned}
$$

with $G^{j}(V, \theta, H)=G_{0}^{j}+G_{\alpha}^{j}(1-\cos 2 \theta)$, where $G_{\alpha}^{j}=$ $A^{j} H / H_{C 2, \perp}+B^{j}$ and $j=S, N$ are superconducting and normal conductance. Solving Eq. (D16) for $H_{C 2}(\theta)$ gives

$$
\begin{aligned}
H_{C 2}(\theta)= & -\frac{1}{2} \frac{H_{C 2, \|}^{2}}{H_{C 2, \perp}} \frac{|\cos \theta|}{\sin ^{2} \theta}+\sqrt{\frac{1}{4} \frac{H_{C 2, \|}^{4}}{H_{C 2, \perp}^{2}} \frac{\cos ^{2} \theta}{\sin ^{4} \theta}+\frac{H_{C 2, \|}^{2}}{\sin ^{2} \theta}} \\
\text { if } \theta \neq 0^{\circ}, 180^{\circ}, & \text { (D18) } \\
& H_{C 2}(\theta)=H_{C 2, \perp} \quad \text { if } \theta=0^{\circ}, 180^{\circ} . \quad \text { (D19) } \\
& \text { APPENDIX E: FITTING TO THE } \\
& \text { PHENOMENOLOGICAL MODEL }
\end{aligned}
$$

\section{APPENDIX E: FITTING TO THE PHENOMENOLOGICAL MODEL}

From the phenomenological model we obtain the fitting

$$
\begin{aligned}
G(V, \theta, H)= & h(\theta)\left\{G_{0}^{N}+\left[A^{N} h\left(0^{\circ}\right)+B^{N}\right](1-\cos 2 \theta)\right\} \\
& +[1-h(\theta)]\left\{G_{0}^{S}+\left[A^{S} h\left(0^{\circ}\right)+B^{S}\right]\right. \\
& (1-\cos 2 \theta)\} .
\end{aligned}
$$

We use the measurements from Fig. 10(b) to assume that $G^{N}=2 G^{S}$ at $V=0$, and by omitting index $S$, we can 
simplify Eq. (E1) as

$$
G(\theta, H)=[1+h(\theta)]\left\{G_{0}+\left[A h\left(0^{\circ}\right)+B\right](1-\cos 2 \theta)\right\},
$$

where $G_{0}, A$, and $B$ need to be found. We apply the experimental values $H_{C 2, \perp}=3.5 \mathrm{kOe}$ and $H_{C 2, \|}=12 \mathrm{kOe}$ and determine $G_{0}$ from $G\left(\theta=0^{\circ}, H\right)$ at $H=0.5 \mathrm{kOe}$ and $H=2 \mathrm{kOe}$ as $G_{0}=5.92375 \mu \mathrm{S}$ and $G_{0}=6.09 \mu \mathrm{S}$, respectively. We use the average of both values $G_{0}=$ $6.0069 \mu \mathrm{S} \approx 6.01 \mu \mathrm{S}$. Using $G\left(\theta=270^{\circ}, H=0.5 \mathrm{kOe}\right)$ and $G\left(\theta=270^{\circ}, H=2 \mathrm{kOe}\right)$ we get a set of two linear equations, which we solve to obtain $A=-0.0415 \mu \mathrm{S}$ and $B=-0.224 \mu \mathrm{S} . G(\theta)$ and $\operatorname{MAAR}(\theta)$ from this phenomenological model are shown in Figs. 9(a)-9(c).

\section{APPENDIX F: $F / I / S$ CONDUCTANCE AT FINITE TEMPERATURE}

We fit the calculated conductance to typical experimental conductance spectra at $H=0$ with in-plane magnetization. We use the extended BTK model as described above and take into account finite temperature $T$ [54]. In the fitting we adjust the barrier height $V_{0}$ and SOC $\alpha$ to match the characteristic properties of the conductance spectra.

A comparison between calculated and measured conductance spectra is shown in Fig. 10. Our emphasis is not to obtain the best possible fits but to recover the main features in the experimental data. For two measured samples,
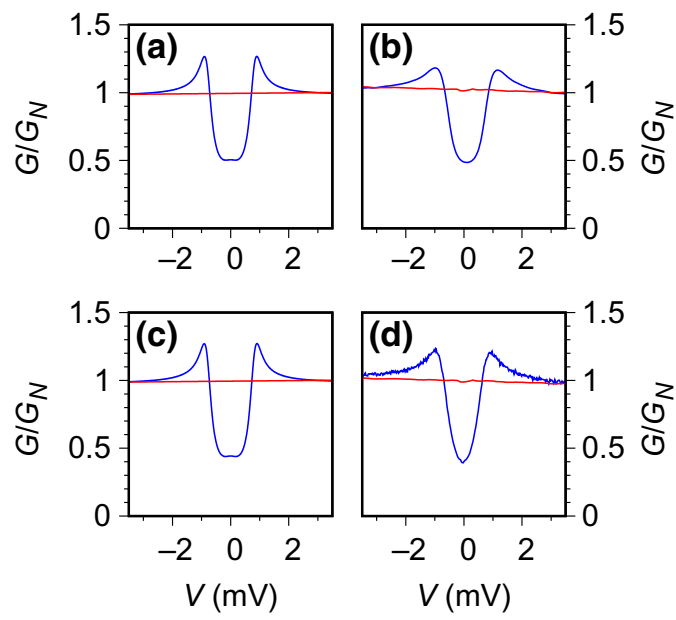

FIG. 10. Calculated and measured $F / I / S$ finite-temperature conductance normalized to $G_{N}=G(3 \mathrm{mV})$ for two different $\mathrm{Fe} / \mathrm{MgO} / \mathrm{V}$ samples at $H=0$. (a) $G / G_{N}$ from the extended BTK model in the superconducting state $(\Delta=0.8 \mathrm{meV}$, blue line) and the normal state $\left(\Delta=0\right.$, red line), $\alpha=4.6 \mathrm{eV}^{2}, Z=0.83, P=$ 0.7 . (b) Measured $G / G_{N}$ in the superconducting state $(T=0.3 \mathrm{~K}$, blue line) and the normal state ( $T=10 \mathrm{~K}$, red line). The sample on which anisotropy measurements from Figs. 4 and. 9 are taken. (c) The same as in (a), but $\alpha=4.2 \mathrm{eV} \AA^{2}$. (d) The same as in (b) for the sample from measurements shown in Fig. 3. the calculated $G(V)$ in Figs. 4(a) and 4(c) differ only in the choice of Rashba SOC, $\alpha$. The ratio of the conductance peak near $V=0.8 \mathrm{mV}$ to the above-the-gap conductance $G / G_{N} \approx 1.2$ suggests that the $\mathrm{Fe} / \mathrm{MgO} / \mathrm{V}$ junction is not in a strong tunneling limit. Consistent with the behavior of epitaxial $\mathrm{MgO}$, we choose $V_{0}=0.3 \mathrm{eV}$ for the effective barrier height and use this value for all results from the extended BTK model at $T=0$ and finite $T$. This corresponds to $Z=V_{0} d \sqrt{m_{F} m_{S}} / \hbar^{2} \sqrt{k_{F} q_{F}}=0.83$, a barrier parameter commonly used in the BTK model $[22,30]$.

[1] I. Žutić, J. Fabian, and S. Das Sarma, Spintronics: Fundamentals and applications, Rev. Mod. Phys. 76, 323 (2004).

[2] S. S. P. Parkin, C. Kaiser, A. Panchula, P. M. Rice, B. Hughes, M. Samant, and S.-H. Yang, Giant tunnelling magnetoresistance at room temperature with $\mathrm{MgO}$ (100) tunnel barriers, Nat. Mater. 3, 862 (2004).

[3] S. Yuasa, T. Nagahama, A. Fukushima, Y. Suzuki, and $\mathrm{K}$. Ando, Giant room temperature magneto-resistance in single-crystal $\mathrm{Fe} / \mathrm{MgO} / \mathrm{Fe}$ magnetic tunnel junctions, Nat. Mater. 3, 868 (2004).

[4] J. Fabian, A. Matos-Abiague, C. Ertler, P. Stano, and I. Žutić, Semiconductor spintronics, Acta Phys. Slov. 57, 565 (2007).

[5] A. Singh, S. Voltan, K. Lahabi, and J. Aarts, Colossal Proximity Effect in a Superconducting Triplet Spin Valve Based on the Half-Metallic Ferromagnet $\mathrm{CrO}_{2}$, Phys. Rev. X 5, 021019 (2015).

[6] J. Linder and J. W. A. Robinson, Superconducting spintronics, Nat. Phys. 11, 307 (2015).

[7] M. Eschrig, Spin-polarized supercurrents for spintronics, Phys. Today 64, 43 (2011).

[8] R. Guerrero, D. Herranz, F. G. Aliev, F. Greullet, C. Tiusan, M. Hehn, and F. Montaigne, High bias voltage effect on spin-dependent conductivity and shot noise in carbon-doped $\mathrm{Fe}(001) / \mathrm{MgO}(001) / \mathrm{Fe}(001)$ magnetic tunnel junctions, Appl. Phys. Lett. 91, 132504 (2007).

[9] F. G. Aliev, R. Guerrero, D. Herranz, R. Villar, F. Greullet, C. Tiusan, and M. Hehn, Very low $1 / f$ noise at room temperature in fully epitaxial $\mathrm{Fe} / \mathrm{MgO} / \mathrm{Fe}$ magnetic tunnel junctions, Appl. Phys. Lett. 91, 232504 (2007).

[10] A. I. Buzdin, Proximity effects in superconductorferromagnet heterostructures, Rev. Mod. Phys. 77, 935 (2005).

[11] F. S. Bergeret, A. F. Volkov, and K. B. Efetov, Odd triplet superconductivity and related phenomena in superconductor-ferromagnet structures, Rev. Mod. Phys. 77, 1321 (2005).

[12] A. A. Golubov, M. Yu. Kupriyanov, and E. Il'ichev, The current-phase relation in Josephson junctions, Rev. Mod. Phys. 76, 411 (2004).

[13] N. Banerjee, J. W. A. Robinson, and M. G. Blamire, Reversible control of spin-polarized supercurrents in ferromagnetic Josephson junctions, Nat. Commun. 5, 4771 (2014).

[14] B. Baek, W. H. Rippard, S. P. Benz, S. E. Russek, and P. D. Dresselhaus, Hybrid superconducting magnetic memory 
device using competing order parameters, Nat. Commun. $\mathbf{5}$, 3888 (2014).

[15] E. C. Gingrich, B. M. Niedzielski, J. A. Glick, Y. Wang, D. L. Miller, R. Loloee, W. P. Pratt, Jr., and N. O. Birge, Controllable $0-\pi$ Josephson junctions containing a ferromagnetic spin valve, Nat. Phys. 12, 564 (2016).

[16] M. Eschrig, in Spintronics Handbook: Spin Transport and Magnetism, edited by E. Y. Tsymbal and I. Žutić (CRC Press, Taylor \& Francis, Boca Raton, FL, 2019), 2nd ed.

[17] J. W. A. Robinson, J. D. S. Witt, and M. G. Blamire, Controlled injection of spin-triplet supercurrents into a strong ferromagnet, Science 329, 59 (2010).

[18] R. S. Keizer, S. T. B. Goennenwein, T. M. Klapwijk, G. Miao, G. Xiao, and A. A. Gupta, A spin triplet supercurrent through the half-metallic ferromagnet $\mathrm{CrO}_{2}$, Nature 439 , 825 (2006).

[19] M. Eschrig, J. Kopu, J. C. Cuevas, and G. Schon, Theory of Half-Metal/Superconductor Heterostructures, Phys. Rev. Lett. 90, 137003 (2003).

[20] L. P. Gorkov and E. I. Rashba, Superconducting 2D System with Lifted Spin Degeneracy Mixed Singlet-Triplet State, Phys. Rev. Lett. 87, 037004 (2001).

[21] F. S. Bergeret and I. V. Tokatly, Singlet-Triplet Conversion and the Long-Range Proximity Effect in SuperconductorFerromagnet Structures with Generic Spin Dependent Fields, Phys. Rev. Lett. 110, 117003 (2013).

[22] P. Högl, A. Matos-Abiague, I. Žutić, and J. Fabian, Magnetoanisotropic Andreev Reflection in FerromagnetSuperconductor Junctions, Phys. Rev. Lett. 115, 116601 (2015).

[23] J. P. Cascales, D. Herranz, F. G. Aliev, T. Szczepanski, V. K. Dugaev, J. Barnas, A. Duluard, M. Hehn, and C. Tiusan, Controlling Shot Noise in Double-Barrier Magnetic Tunnel Junctions, Phys. Rev. Lett. 109, 066601 (2012).

[24] I. Žutić, A. Matos-Abiague, B. Scharf, H. Dery, and K. D. Belashchenko, Proximitized materials, Mater. Today 22, 85 (2019).

[25] K. D. Belashchenko and E. Y. Tsymbal, in Handbook of Spin Transport and Magnetism, edited by E. Y. Tsymbal and I. Žutić (CRC Press, Boca Raton, FL, 2012).

[26] P. Blaha, K. Schwarz, G. K. H. Madsen, D. Kvasnicka, and J. Luitz, WIEN2k, An Augmented Plane Wave + Local Orbitals Program for Calculating Crystal Properties (TU Vienna, Vienna, 2001).

[27] C. Tiusan, M. Hehn, F. Montaigne, F. Greullet, S. Andrieu, and A. Schuhl, Spin tunneling phenomena in single crystal magnetic tunnel junction systems, J. Phys.: Condens. Matter 19, 165201 (2007).

[28] W. H. Butler, X.-G. Zhang, T. C. Schulthess, and J. M. MacLaren, Spin-dependent tunneling conductance of $\mathrm{Fe}-\mathrm{MgO}-\mathrm{Fe}$ sandwiches, Phys. Rev. B 63, 054416 (2001).

[29] I. Martínez, C. Tiusan, M. Hehn, M. Chshiev, and F. G. Aliev, Symmetry broken spin reorientation transition in epitaxial $\mathrm{MgO} / \mathrm{Fe} / \mathrm{MgO}$ layers with competing anisotropies, Sci. Rep. 8, 9463 (2018).

[30] G. E. Blonder, M. Tinkham, and T. M. Klapwijk, Transition from metallic to tunneling regimes in superconducting microconstrictions: Excess current, charge imbalance, and supercurrent conversion, Phys. Rev. B 25, 4515 (1982).
[31] B. Soulen, Jr., J. M. Byers, M. S. Osofsky, B. Nadgorny, T. Ambrose, S. F. Cheng, P. R. Broussard, C. T. Tanaka, J. Nowak, J. S. Moodera, A. Barry, and J. M. D. Coey, Measuring the spin polarization of a metal with a superconducting point contact, Science 282, 85 (1998).

[32] J. S. Parker, S. M. Watts, P. G. Ivanov, and P. Xiong, Spin Polarization of $\mathrm{CrO}_{2}$ at and across an Artificial Barrier, Phys. Rev. Lett. 88, 196601 (2002).

[33] B. E. Nadgorny, in Handbook of Spin Transport and Magnetism, edited by E. Y. Tsymbal and I. Žutić (CRC Press, Boca Raton, FL, 2012).

[34] C. Ren, J. Trbovic, R. L. Kallaher, J. G. Braden, J. S. Parker, S. von Molnár, and P. Xiong, Measurement of the spin polarization of the magnetic semiconductor EuS with zerofield and Zeeman-split Andreev reflection spectroscopy, Phys. Rev. B 75, 205208 (2007).

[35] Y. Miyoshi, Y. Bugoslavsky, and L. F. Cohen, Andreev reflection spectroscopy of niobium point contacts in a magnetic field, Phys. Rev. B 72, 012502 (2005).

[36] S. Sangiao, J. M. De Teresa, M. R. Ibarra, I. Guillamon, H. Suderow, S. Vieira, and L. Morellon, Andreev reflection under high magnetic fields in ferromagnet-superconductor nanocontacts, Phys. Rev. B 84, 233402 (2011).

[37] K. A. Yates, M. S. Anwar, J. Aarts, O. Conde, M. Eschrig, and T. Lofwander and L. F. Cohen, Andreev spectroscopy of $\mathrm{CrO}_{2}$ thin films on $\mathrm{TiO}_{2}$ and $\mathrm{Al}_{2} \mathrm{O}_{3}$, EPL 103, 67005 (2013).

[38] I. Žutić and S. Das Sarma, Spin-polarized transport and Andreev reflection in semiconductor/superconductor hybrid structures, Phys. Rev. B 60, 16322(R) (1999).

[39] A. Vansteenkiste, J. Leliaert, M. Dvornik, M. Helsen, F. Garcia-Sanchez, and B. Van Waeyenberge, The design and verification of mumax3, AIP Adv. 4, 107133 (2014).

[40] N. E. Alekseevskii, V. M. Sakosarenko, K. Blüthner, and H. J. Köhler, Superconducting properties of vanadium films, Phys. Status Solidi A 34, 541 (1976).

[41] T. Sekula and R. H. Kernohan, Magnetic properties of superconducting vanadium, Phys. Rev. B 5, 904 (1972).

[42] M. Tinkham, Effect of fluxoid quantization on transitions of superconducting films, Phys. Rev. 129, 2413 (1963).

[43] C. Visani, Z. Sefrioui, J. Tornos, C. Leon, J. Briatico, M. Bibes, A. Barthélémy, J. Santamaría, and E. Villegas, Equal-spin Andreev reflection and long-range coherent transport in high-temperature superconductor/half-metallic ferromagnet junctions, Nat. Phys. 8, 539 (2012).

[44] M. J. M. de Jong and C. W. J. Beenakker, Andreev Reflection in Ferromagnet-Superconductor Junctions, Phys. Rev. Lett. 74, 1657 (1995).

[45] K.-R. Jeon, C. Ciccarelli, A. J. Ferguson, H. Kurebayashi, L. F. Cohen, X. Montiel, M. Eschrig, J. W. A. Robinson, and M. G. Blamire, Enhanced spin pumping into superconductors provides evidence for superconducting pure spin currents, Nat. Mater. 17, 499 (2018).

[46] N. Banerjee, J. A. Ouassou, Y. Zhu, N. A. Stelmashenko, J. Linder, and M. G. Blamire, Controlling the superconducting transition by spin-orbit coupling, Phys. Rev. B 97, 184521 (2018).

[47] L. G. Johnes, N. Banerjee, and J. Linder, Magnetization reorientation due to the superconducting transition 
in heavy-metal heterostructures, Phys. Rev. B 99, 134516 (2019).

[48] The competence 'Deposit and Analysis of Nanomaterials under Ultra-High Vacuum' center (DAUM) within the Jean Lamour Institute, Nancy, France is based on an innovative equipment (the TUBE) allowing the inter-connexion under ultra-high vacuum of multiple complex tools for elaboration, analysis and nano-structuration of materials nanometre scale (https://ijl.univ-lorraine.fr/en/research/competence -centers/deposit-and-analysis-of-nanomaterials-under-ultra -high-vacuum-daum/).

[49] Ya. M. Blanter and M. Büttiker, Shot noise in mesoscopic conductors, Phys. Rep. 336, 1 (2000).

[50] T. Szczepański, V. K. Dugaev, J. Barnaś, J. P. Cascales, and F. G. Aliev, Shot noise in magnetic double-barrier tunnel junctions, Phys. Rev. B 87, 155406 (2013).
[51] P. G. De Gennes, Superconductivity of Metals and Alloys (Addison-Wesley, Reading, MA, 1989).

[52] A. Matos-Abiague and J. Fabian, Anisotropic tunneling magnetoresistance and tunneling anisotropic magnetoresistance: Spin-orbit coupling in magnetic tunnel junctions, Phys. Rev. B 79, 155303 (2009).

[53] M. Wimmer, M. Lobenhofer, J. Moser, A. Matos-Abiague, D. Schuh, W. Wegscheider, J. Fabian, K. Richter, and D. Weiss, Orbital effects on tunneling anisotropic magnetoresistance in Fe/GaAs/Au junctions, Phys. Rev. B 80, 121301 (2009).

[54] T. Hirai, Y. Tanaka, N. Yoshida, Y. Asano, J. Inoue, and S. Kashiwaya, Temperature dependence of spinpolarized transport in ferromagnet/unconventional superconductor junctions, Phys. Rev. B 67, 174501 (2003). 